\title{
Microbial invasion of a toxic medium is facilitated by a resident community but inhibited as the community co-evolves
}

\author{
Philippe Piccardi ${ }^{1}$, Géraldine Alberti ${ }^{1}$, Jake M. Alexander ${ }^{2}$, and Sara Mitri ${ }^{1,3, \mathbb{}}$ \\ ${ }^{1}$ Département de Microbiologie Fondamentale, Université de Lausanne, 1015 Lausanne, Switzerland \\ ${ }^{2}$ Department of Environmental Systems Science, ETH Zurich \\ ${ }^{3}$ Swiss Institute of Bioinformatics
}

\begin{abstract}
Predicting whether microbial invaders will colonize 45 an environment is critical for managing natural and engineered ${ }_{46}$ ecosystems, and controlling infectious disease. Invaders often ${ }_{47}$ face competition by resident microbes. But how invasions play out in communities dominated by facilitative interactions is less clear. We previously showed that growth medium toxicity can promote facilitation between four bacterial species, as species ${ }^{50}$ that cannot grow alone rely on others to survive. Following 51 the same logic, here we allowed other bacterial species to in- 52 vade the four-species community, and found that invaders could 53 more easily colonize a toxic medium when the community was 54 present. In a more benign environment instead, invasive species that could survive alone colonized more successfully when the residents were absent. Next, we asked whether early colonists could exclude future ones through a priority effect, by inoculating the invaders into the resident community only after its members had co-evolved for $\mathbf{4 4}$ weeks. Compared to the ances- ${ }^{5}$ tral community, the co-evolved resident community was more ${ }^{60}$ competitive toward invaders, and less affected by them. Our ${ }^{6}$ experiments show how communities may assemble by facilitating one another in harsh, sterile environments, but that arriving 63 after community members have co-evolved can limit invasion success.
\end{abstract}

Evolution | Community Monopolization Hypothesis | priority effects | invasion | bacterial community | facilitation | competition | Stress Gradient Hypothesis 67

Correspondence: sara.mitri@unil.ch

\section{Introduction}

Successful colonization of invader microorganisms into ster- 72 ile environments or existing microbial communities are com- 73 mon, and can impact ecosystem diversity and function, po- 74 tentially with dramatic consequences (1-3). A better under- 75 standing of the factors driving microbial invasions may help 76 to prevent the spread and establishment of invasive species, 77 or to aid the intentional introduction of a new species for a 78 desired purpose. For example, it might be desirable to pre- 79 vent the invasion of a species that reduces the efficiency of 80 a bioremediation system (4), or to promote the colonization 81 of probiotic species in the intestinal microbiome of a patient 82 $(5,6)$.

What determines the ability of an invasive species to colo- 84 nize an existing ecosystem depends on the characteristics of 85 both the invading species and the resident community $(7,8)$. 86 Many theoretical and empirical studies have established fac- 87 tors that influence invasion outcome, such as propagule pressure (9-14), resident community productivity (15), genotypic richness of invaders $(13,16)$ or the resident community $(3,13,17-20)$, community niche coverage $(3,21,22)$ and abiotic conditions (e.g. the presence of antibiotics (23)).

Invasion success may also depend on the sign and strength of interactions between resident community members, and between residents and invaders (24). Previous studies tend to find that invaders compete with resident species $(3,13$, $17,20,23,25,26)$, which is consistent with competition typically dominating microbial communities (27-29). However, in sterile environments, early colonizers can facilitate the arrival of other species (30-35). This occurs when new communities assemble and groups of species follow one another in so-called "successions", for example in the formation of dental plaque $(36,37)$ or marine particle communities $(32,33)$. Facilitation likely occurs in newly assembling communities, as sterile environments are typically difficult to colonize, for example if they have an extreme $\mathrm{pH}$, contain toxic compounds, or are lacking in easily accessible nutrients or water. Pioneer species may alter the environment in ways that facilitate invasion by new species that would otherwise not survive $(24,38-41)$. This is in line with the Stress Gradient Hypothesis (SGH), which predicts that species are more likely to interact positively in stressful environments (42-48). The link between the SGH and microbial invasion has, however, not yet been experimentally tested.

As more species colonize the environment and species diversity increases, previously available niches begin to fill up, such that competition is expected to increase and invasion success to drop. The negative relationship between invasion success and species richness and diversity have been wellestablished $(3,13,17-20)$. As time passes, resident species may co-evolve to reduce niche overlap and availability in a way that would prevent further invasion. The Community Monopolization Hypothesis predicts that early colonisers adapt to use available resources efficiently, yielding a competitive advantage against later-arriving species (49-52), also known as a "priority effect" $(31,53)$. One may also expect such co-evolved resident species to be less perturbed by species invasion (49). Experimentally disentangling the role of the different factors discussed above on invasion success and resistance can be challenging. 
Here we aim to test the effect of the two less-well under- 144 stood factors (the SGH and priority effects) on bacterial in- 145 vasion success by studying invasion into a synthetic bacte- ${ }_{146}$ rial community whose composition is fixed at four species: ${ }_{147}$ Agrobacterium tumefaciens, Comamonas testosteroni, Mi- 148 crobacterium saperdae and Ochrobactrum anthropi. These ${ }_{149}$ four species can grow and bioremediate Metal Working Flu- 150 ids (MWF) (47, 54), an industrial fluid used in metal manu- 151 facturing. MWFs contain mineral oils, emulsifiers and bio- 152 cides, some of which are toxic to bacteria. In previous work 153 (47), we showed that when the four species were grown to- 154 gether in this toxic environment, they facilitated each other's 155 survival compared to when they were alone. Instead, when 156 we added amino acids to make the environment more permissive, competition between species increased. This sys- 157 tem allows us to study biological invasion while experimen- 158 tally manipulating environmental conditions to control inter- 159 actions between community members and holding all other 160 factors constant. Another advantage of this system is that it 161 stabilizes over evolutionary time-scales, allowing us to ex- 162 plore the effect of community co-evolution on microbial in- 163 vasion.

Using four invader species, Aeromonas caviae, Klebsiella 165 pneumoniae, Providencia rettgeri, and Pseudomonas fulva 166 that were isolated from waste MWF (chosen from a set of 167 20 based on our ability to distinguish them from the resident 168 species), we first show that the resident community facili- 169 tates invasion of species that cannot grow alone, but inhibits 170 those that can. Whether or not species could grow alone was 171 modulated by changes in the growth medium, in particular 172 the addition of amino acids as nutrient supply. Second, after ${ }^{173}$ co-evolving the four resident species for 44 weeks, we found 174 that invasions were still possible in MWF, but the growth of 175 the invaders was inhibited relative to the ancestral commu- 176 nity and the co-evolved resident species were less affected by invasions. Together, our results show that facilitative com- ${ }^{177}$ munities are easier to invade than competitive ones, but that a ${ }^{178}$ co-evolved community is more robust to invasion compared ${ }^{179}$ to an ancestral one.

\section{Results}

The resident community facilitates the invasion of ${ }_{184}$ species that cannot grow alone. We first ask whether the ${ }_{185}$ four invader species (A. caviae, K. pneumoniae, P. rettgeri, ${ }_{186}$ and $P$. fulva), could individually colonize MWF and to what ${ }_{187}$ extent the resident community promotes or inhibits inva- 188 sion. The resident community was cultured in MWF for $1_{189}$ week, at the end of which $1 \%$ of the population was trans- 190 ferred into fresh media, and this was repeated for a total of $4{ }_{191}$ weeks. Each invader species was inoculated into three repli- 192 cate microcosms of the resident community 48 hours after 193 the first transfer, presumably during the community's expo- 194 nential growth phase (Fig. S1A). The resident community 195 was always invaded by a single invader species at a time. 196 As a control treatment, we inoculated each of the invader ${ }_{197}$ species into sterile MWF and performed transfers in paral- 198 lel (Fig. S1B). The abundance of the invading and resident 199 species was quantified at inoculation and before each transfer (invader species in Fig. 1A; resident species in Fig. S2A, left).

P. fulva was the only one of the four invader species that could colonize the MWF when alone (Fig. 1A (red dotted line), C). Instead, when the community was present, the number of successful invasions increased: A. caviae, K. pneumoniae and $P$. fulva colonized the MWF containing the resident community, while $P$. rettgeri still did not (Fig. 1A (red solid line), C). These results are in line with our previous findings (47) that species that cannot grow alone in MWF are likely to be facilitated by other species, explaining why in some cases invasion is only successful when the community is present.

Residents inhibit invaders that can grow alone in a more permissive medium. MWFs are designed to prevent bacterial contamination and are therefore quite toxic. This explains why only one of the invader species was able to grow alone in the MWF medium. To explore invasion in a less harsh environment, we enriched the medium by adding $1 \%$ casamino acids (MWF+AA). Casamino acids are a nutrient source for 3 out of the 4 resident community members and, according to previous work $(47,55)$, we expect more negative interactions in a more permissive medium. We found that $K$. pneumoniae, $P$. fulva and $P$. rettgeri could colonize $\mathrm{MWF}+\mathrm{AA}$ alone, while $A$. caviae still suffered from the environmental toxicity (invader species Fig. 1B, C; resident species Fig. S2A, right). The three species that were able to colonize alone were still able to invade the community, but significantly less well compared to when the community was absent (Kruskal-Wallis, all p-values $<0.05$, Fig. 1C). Consistent with previous work (47), our results suggest that in this more permissive environment, the community competes with the invaders.

\section{A resident community co-evolved in MWF is more} competitive toward invaders. The capacity to colonize a resident community might depend on community history: resident species that have adapted to one another in a given environment may be more likely to exclude future colonists through a priority effect (49-51). To test this hypothesis, we extend the pre-invasion phase to 44 weeks, allowing the four resident species to adapt to MWF and to each other (see Material and Methods). Next, we mixed one co-evolved isolate of each species and call this the "co-evolved community" (Fig. S1C).

We now ask to what extent the invader species can colonize the co-evolved resident community compared to the ancestral one. We found that while $P$. rettgeri could colonize neither, $A$. caviae, K. pneumoniae and $P$. fulva colonized both the ancestral and co-evolved communities (invader species Fig. 2A, B; resident species Fig. S2B). However, all three invader species had a smaller invasion magnitude in the co-evolved compared to the ancestral community (Kurskal-Wallis, A. caviae p-value $<0.0005, K$. pneumoniae p-value $<0.0005, P$. fulva p-value $<0.05$, Fig. 2B). The invasion outcome for $A$. caviae was initially inconclusive, where in one out of two biological replicates the invader went extinct when inoculated into the 

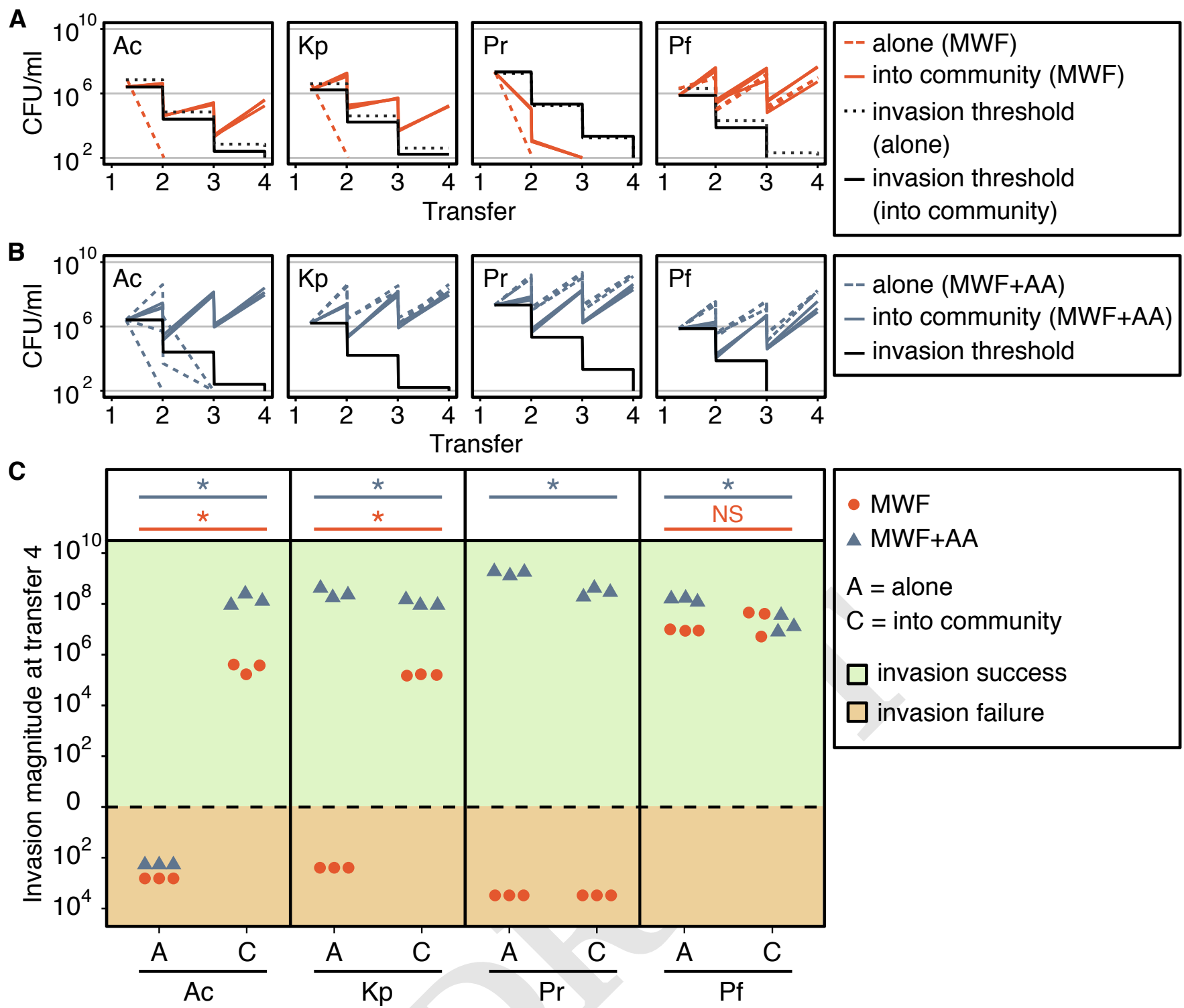

Fig. 1. Outcome of invasion in MWF or MWF+AA. (A) Abundances of the four invader species in MWF at different transfers, quantified at first inoculation and before each transfer. The four invader species were grown alone or inoculated into the growing resident community after it had been transferred once and until transfer 4 (1 transfer every 7 days). At each transfer, the culture is diluted 100-fold. The experiments were done in parallel except for the invader alone, which was done separately. The black dotted line and the black solid line represent the invasion thresholds of the four invader species alone and into the community, respectively, which represents the abundance of a non-growing invader over time. We count an invasion as successful if the abundance of the invader at the end of the transfers is higher than the invasion threshold. (B) Abundances of the four invader species in MWF+AA at different transfers. The black solid line represents the invasion threshold when grown alone "A" or invaded into the community "C". The experiments were all conducted in parallel. (C) To exclude the effect of the choice of dilution rate on invasion success, we calculate the "invasion magnitude": the abundance of each invader at transfer 4 minus its corresponding invasion threshold. If this number is higher or lower than zero, then the invasion is successful $(+)$ or failed (-), respectively. From left to right: $A$. caviae $(\mathrm{Ac}), K$. pneumoniae $(\mathrm{Kp}), P$. rettgeri $(\mathrm{Pr}), P$. fulva $(\mathrm{Pf})$. Statistical significance is marked above the data points (P-values: ${ }^{*}<0.05, \mathrm{NS}=$ not significant). For abundance of resident species see Fig. S2.

ancestral community (Fig. S3A, B). We tested whether this 212 was due to variability in propagule pressure (13), but found ${ }_{213}$ no evidence for this, as different invasion population sizes of ${ }_{214}$ A. caviae all converged to a similar population size at trans- 215 fer 4 (invader species Fig. S4; resident species Fig. S5). We 216 therefore concluded that the death of A. caviae in one biolog- 217 ical replicate might have been due to a technical error (Fig. 218 S3).

219 220 We next wondered whether the pattern observed for $A_{221}{ }_{221}$ caviae, $K$. pneumoniae and $P$. fulva was specific to these ${ }_{222}$ invader species colonizing our community of four resident ${ }_{223}$ species. One way to explore this is to exclude one species from the co-evolutionary process and allow it to invade at a later stage. We did this by co-evolving three of the resident species, A. tumefaciens, C. testosteroni and M. saperdae together in MWF for 44 weeks, excluding $O$. anthropi. Next, we combined single isolates of the three co-evolved species and invaded the wild-type $O$. anthropi into this co-evolved 3species community (Fig. S1D). As before, $O$. anthropi could not colonize the MWF when alone (as in (47), Fig. 2C), but invaded successfully when inoculated into the ancestral or the co-evolved community of three. Consistent with the previous invasion assays (Fig. 2A, B), and our hypothesis that a coevolved community is more difficult to invade, $O$. anthropi 

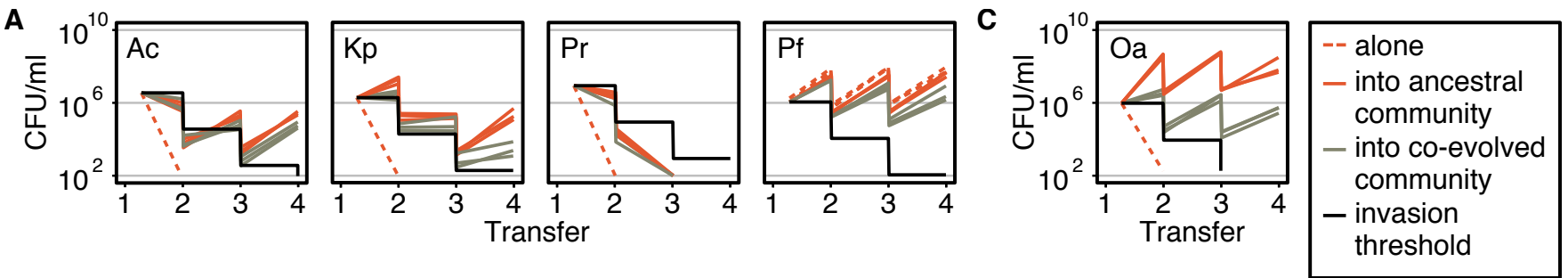

B
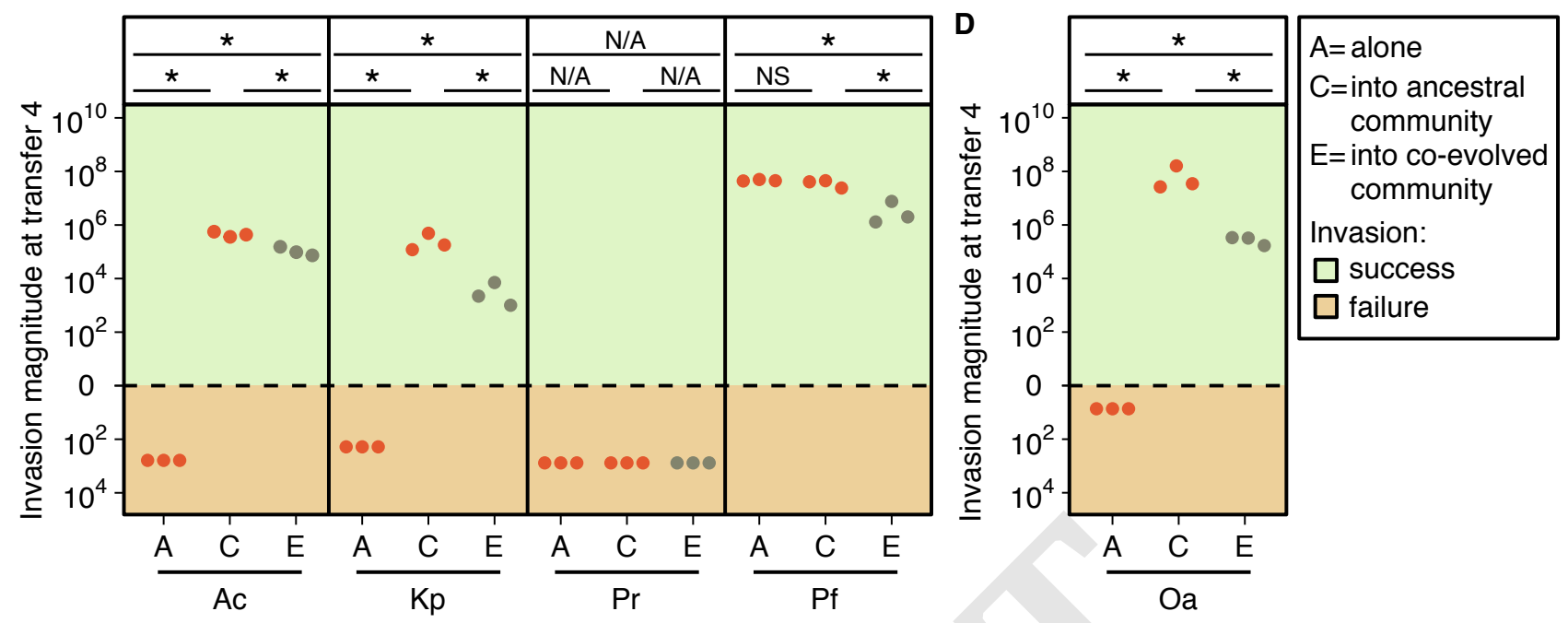

Fig. 2. Invasion into ancestral or co-evolved communities. (A, C) Invader species were grown alone, or inoculated into the ancestral or the co-evolved community in MWF. Cultures were diluted 100 -fold in fresh MWF every 7 days for a total of 4 transfers. The experiments were all conducted in parallel. The black line represents the invasion threshold. (B, D) Invasion magnitude (abundance at transfer 4 minus the invasion threshold) alone "A", into the ancestral community " $\mathrm{C}$ " or into the evolved community " $\mathrm{E}$ ". Positive or negative invasion magnitudes indicate successful (+) or failed (-) invasions, respectively. From left to right: $A$. caviae (Ac), K. pneumoniae (Kp), P. rettgeri (Pr), P. fulva (Pf), O. anthropi (Oa). Statistical significances are marked above the data points (P-values: ${ }^{*}<0.05$, NS $=$ not significant, N/A = not applicable statistic). See Fig. 1 caption for more details. In panels $A$ and $B$, the resident community consists of a co-culture of four ancestral or evolved clonal populations, while in panels $C$ and $D$, the community contained only three clonal populations (four residents, without $O$. anthropi), either ancestral or evolved. For abundance of the four-species or three-species resident communities see Fig. S2B or Fig. S6, respectively.

grew significantly worse when it was inoculated into the co- 249 evolved 3-species community compared to the correspond-250 ing ancestral one (invader O. anthropi Fig. 2C, D; resident 251 species Fig. S6).

In sum, while invasions into a community co-evolved in ${ }^{253}$ MWF are still possible, co-evolved community members in- 254 hibit invading species more than their ancestors.

\section{Co-evolved communities are less affected by invasion compared to their ancestors. So far, we have focused on} the effect of the resident community on the invading species. ${ }^{250}$ Next, we consider how robust the resident community is to ${ }^{260}$ these invasion events.

The abundance of each community member was quantified ${ }^{262}$ at the beginning of the experiment and before each transfer. Here, we focus on their abundance at transfer 4, representing cumulative effects (but see e.g. Fig. S2 for remaining ${ }_{260}$ data). In all our treatments, the four resident species were maintained over the four transfers. The abundance of two of the ancestral resident species, A. tumefaciens and $O$. anthropi, was significantly lower when invaded by $P$. fulva (ttest, both p-values $<0.005$, Fig. 3A, Fig. S7A). Otherwise, ${ }^{269}$ we detected no significant changes in their abundance follow- 270 ing invasion by other species. This lack of perturbation was 271 also observed for $C$. testosteroni. M. saperdae's abundance 272 was instead greater in the presence of most invaders (t-test, 273
A. caviae and $P$. rettgeri both $\mathrm{p}$-values $<0.005$, P. fulva $\mathrm{p}$ value $<0.005$, Fig. 3A, Fig. S7A). This is not surprising, as we know that $M$. saperdae strongly depends on other species to grow in MWF (47).

Once the community had co-evolved, the abundances of $A$. tumefaciens and $M$. saperdae were no longer significantly affected by the invasion of $P$. fulva (Fig. 3B). The abundance of $O$. anthropi was still lower following invasion by P. fulva, but significantly less compared to the ancestor (ancestor versus co-evolved, t-test, p-value $=0.0167$, Fig. 3A, $\mathrm{B}$, last column). In addition, the abundance of $M$. saperdae was no longer significantly positively affected by any of the invaders. This may be because the co-evolved M. saperdae grows significantly better within the resident community (Fig. 3A, B). The co-evolved 3-species community behaved similarly: While the abundance of ancestral A. tumefaciens was significantly lower following the invasion of $O$. anthropi (t-test, p-value $<0.05$ ), its co-evolved counterpart was not (Fig. 3C, D). Altogether, co-evolved resident communities were more robust to invasion compared to ancestral ones.

\section{Discussion}

Studies on microbial invasion often focus on how resident community composition and species richness affect invasion outcomes. Here, we chose instead to work with a resident community whose composition was fixed at the same four 
bioRxiv preprint doi: https://doi.org/10.1101/2022.03.03.482806; this version posted March 3, 2022. The copyright holder for this preprint (which was not certified by peer review) is the author/funder, who has granted bioRxiv a license to display the preprint in perpetuity. It is made available under aCC-BY 4.0 International license.

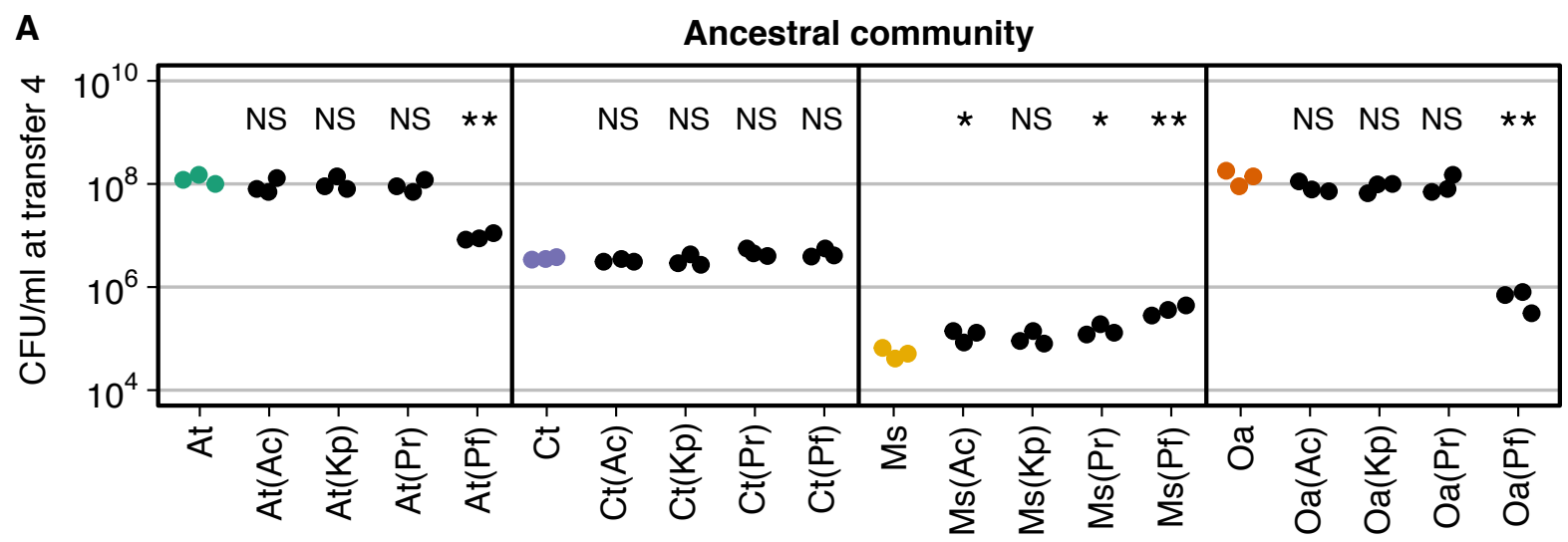

B

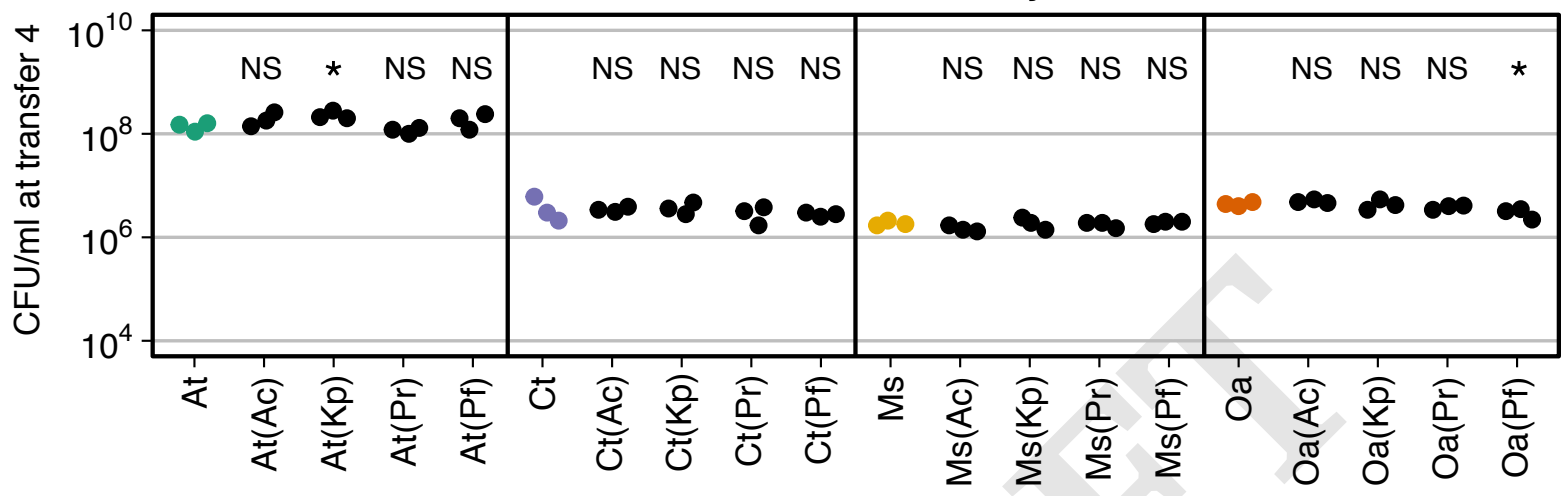

C

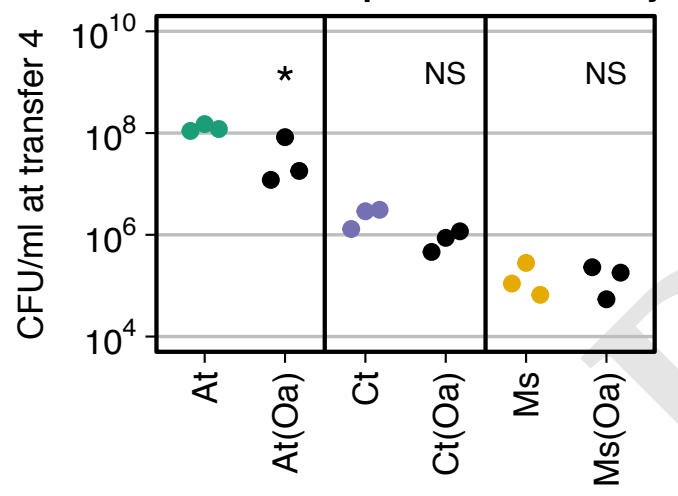

D

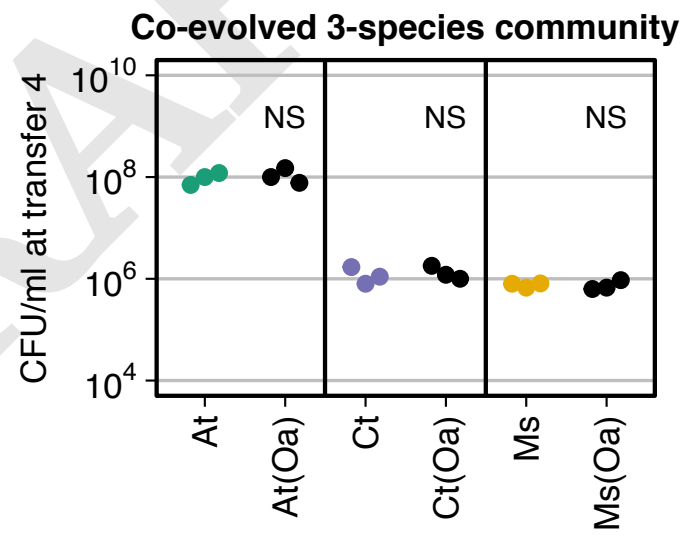

Fig. 3. Bacterial abundance of ancestral or co-evolved community members with or without invader. Each panel represents the total population size (CFU/mL) at transfer 4 of a resident member in the ancestral community (A), the co-evolved community (B), the ancestral 3-species community (C) and the co-evolved 3-species community (D). The full datasets are in Fig. S2 and S6. All experiments were performed in MWF. The bacterial abundance of community members without any invader species is represented by colored dots, and once invaded by black dots (invader species indicated in brackets). From left to right: $A$. tumefaciens (At), $C$. testosteroni (Ct), M. saperdae (Ms), O. anthropi (Oa), A. caviae (Ac), K. pneumoniae (Kp), P. rettgeri ( $\mathrm{Pr}), P$. fulva (Pf). We compared the data points of each species when invaded to the corresponding data points when co-cultured with other community members but without invasion. Statistical significance is marked above the data points $\left(\mathrm{P}\right.$-values: ${ }^{\star}<0.05$, ${ }^{* *}<0.01, \mathrm{NS}=$ not significant).

species, and ask how their environment - specifically envi- 285 ronmental harshness - and their common evolutionary his- 286 tory affect invasion success.

By increasing the permissiveness of a harsh medium (MWF) ${ }^{288}$ through the addition of amino acids (MWF+AA), the number ${ }^{289}$ of invader species able to grow alone increased from a single 290 one to 3 out of 4 species. In almost all cases where invaders 291 died alone, the resident community facilitated their survival ${ }^{292}$ and growth. Instead, if invaders could survive alone in the ${ }^{293}$ more permissive environment, they experienced a net nega- ${ }^{294}$ tive effect if the community was present. This is consistent ${ }^{295}$ with our previous research (47), and more generally with the Stress Gradient Hypothesis (SGH), which is rarely linked to invasion ecology. Our results highlight this intuitive link: in a harsh environment colonized by few species, invasion success may be high, as niches are still available and invaders can rely on the presence of the residents to survive. This is expected if early-arriving species improve the environment, facilitating the growth of others that are less well adapted to it (38-41). Alternative dynamics are possible if the first colonizers alter the environment in a way that strongly inhibits future invaders (31), or if late colonizers out-compete earlier 
ones and replace them (56), but this is not what we observe ${ }_{353}$ in our system.

If this intuition is correct, it would explain why the assem- ${ }^{355}$ bly of many natural microbial communities is often highly ${ }^{356}$ predictable $(32,33,36,37,57)$. For example, microbial col- ${ }^{357}$ onization of the healthy mammalian gut displays specific pat- ${ }^{358}$ terns of species arrival $(57,58)$. But is a sterile gut a harsh ${ }^{359}$ environment? Microbes colonizing a newborn gut must sur- 360 vive the acidic conditions of the stomach, the host's immune ${ }^{36}$ system and bile acids and cholesterol produced by the host 362 that are toxic for most microbial species (59). A few spe- 363 cialized Lactobacillus and Bifidobacterium species produce 364 bile resistance proteins (60), which allow them to colonize 365 the gut and may facilitate the arrival of other species (61). 366 Similar dynamics may occur in other systems where strong ${ }^{367}$ ecosystem perturbations clear the ground for new communi- 368 ties to assemble, such as following antibiotic treatments, or 369 the heavy pollution of soils. However, it remains to be seen 370 whether first colonizers facilitate future arrivals as would be ${ }^{37}$ predicted by the $\mathrm{SGH}(42,47,62)$, or whether it is more of a 372 race to fill available niches.

Once a community has assembled despite the challenging en- ${ }^{374}$ vironment, we next asked whether the timing of invasion mat- ${ }^{375}$ ters. In our experiments, early invaders fared better than those 376 colonizing a community whose species had co-evolved. But 377 what makes the co-evolved MWF community more resistant 378 and robust to invasion? We noticed that during the last of 379 the 44 transfers, there were relatively small population size 380 fluctuations compared to the first transfers (Fig. S8A, B), ${ }_{381}$ suggesting that ecological dynamics had stabilized, which is 382 expected based on similar studies (63). Community stability ${ }^{38}$ is often defined as the capacity of a community to return to its 384 initial state after perturbation, such as an invasion (64-67). It 385 is then perhaps not surprising that ecological stability corre- 386 lated with invasion resistance and robustness (Fig. 3A, B or ${ }_{387}$ C, D).

The higher stability - or the higher invasion resistance - of ${ }^{389}$ co-evolved communities can be due to several reasons. First, ${ }^{390}$ co-evolved A. tumefaciens and M. saperdae (but not C. testos- 391 teroni and $O$. anthropi) grew to larger population sizes during 392 the first days (Fig. S9), suggesting that they may take up nu- ${ }^{39}$ trients faster, making them competitive against invaders. Sec- 394 ond, communities with high strain-level diversity tend to be ${ }^{395}$ more productive and more robust against invasions $(15,68) .{ }^{396}$ However, strain-level diversity and community productivity ${ }^{397}$ cannot explain invasion outcomes in our system: by using 398 single isolates from the co-evolved communities, our exper- 399 iments had the same strain-level diversity in all treatments ${ }^{400}$ (Fig. S1A, C); we also observed no significant differences in ${ }^{401}$ productivity between ancestral and co-evolved communities ${ }^{402}$ (Fig. S10). Third, it could be that the residents co-evolved ${ }^{403}$ to actively inhibit other species. If this were true, we might ${ }^{404}$ expect the resident species to interact negatively with one an- 405 other, but on measuring pair-wise interactions between an- 406 cestral (Fig. S11G) and co-evolved species (Fig. S12G, Fig. 407 S13F) we only observed that positive interactions weakened 408 between A. tumefaciens and C. testosteroni, and increased 409 in the other two species (Fig. S12G, Fig. S13G), making this scenario less plausible. Finally, the co-evolving residents may have partitioned the available niches among themselves, leaving little "space" for new arrivals (31). Further investigation, possibly using metabolomics analyses, would be needed to clarify whether this is the case and to more mechanistically understand microbial resistance against invasion.

Our fixed-species experimental design revealed some interesting patterns of invasion success, but also has its limitations. One confounding factor is that adding amino acids to the growth medium allowed more species to grow alone, but also provided new niches for invader species to occupy. This is reflected in the higher overall invasion magnitude of species in MWF+AA compared to MWF (Fig. 1C). But despite these additional niches, invasion magnitude was still lower when the community was present compared to its absence (Fig. 1C). This made it difficult to interpret how invaders affected the resident community grown in MWF+AA: the effects varied depending on the invader species and the resident species with no clear pattern (Fig. S7). Digging deeper into the mechanisms behind the interactions in our system, and developing a theoretical basis for what to expect may help to understand these effects.

One could also question whether a small synthetic community is representative of natural communities and their diversity. A mathematical model in our previous study indicated that competition would increase with a higher number of species in MWF (47), and perhaps we would expect invasions to be less successful in this context. This would also align with experiments involving larger communities that presumably occupy more niches and leave fewer resources for the invader $(13,17-20,25)$. Nevertheless, our community could help to understand the first phases of community assembly, when only few species have colonized.

Another weakness of our study is the arbitrary choice to perform four transfers at a $1 \%$ dilution rate. To compensate, we were careful to define our measures independently of these choices, such that we could compare between treatments rather than considering absolute measures of invasion. We quantified "invasion success", representing absolute population increase or decrease, and "invasion magnitude", which compares population sizes between treatments at the end of the experiment. Another possibility would have been to extend the length of the experiment to observe whether invaders eventually went extinct or established themselves. However, as we were interested in the ecological dynamics of invasion separately from the evolutionary dynamics of the resident community, we decided to keep the invasion time-scale short and assume that species' genetic adaptation to the environment and each other was negligible. In reality, of course, invaders might acquire mutations that increase invasion success.

In conclusion, we used a model system to disentangle interactions between species and measure their effect on microbial invasion. This revealed that a small, facilitative resident community can improve the environment for species that would otherwise be unable to colonise. However, a com- 
munity whose residents have adapted to the environment and 466 each other is more difficult to invade. Our work links invasion ${ }_{467}$ ecology with the SGH, priority effects and historical contin- 468 gency $(42,49,69)$. We provide a fresh perspective on com- 469 munity assembly as a sequence of invasion events into a harsh 470 environment, where facilitation may be dominant at first as 471 species complement each other, but decreases as niches are ${ }_{472}$ occupied through co-evolution.

\section{Materials and Methods}

Study system. The 4 bacterial species used to assemble the resident community were isolated from MWF (54), and are referred to as: Agrobacterium tumefaciens str. MWF001, $\mathrm{Co}^{-}{ }_{479}$ mamonas testosteroni str. MWF001, Microbacterium saper- ${ }_{480}$ dae str. MWF001 and Ochrobactrum anthropi str. MWF001 (as in (47)). The additional four bacterial species, used to invade the resident community, were kindly donated by $\mathrm{Pe}-{ }_{483}$ ter Küenzi from Blaser Swisslube AG, Hasle-Rüegsau, and ${ }_{484}^{48}$ we refer to them as: Aeromonas caviae str. Blaser001, Klebsiella pneumoniae str. Blaser001, Providencia rettgeri str. Blaser001, and Pseudomonas fulva str. Blaser001. The Metal-Working Fluid (MWF, Castrol Hysol XF, acquired in 2016) was prepared at a concentration of $0.5 \%(\mathrm{v} / \mathrm{v})$, diluted in water with the addition of selected salts and metal traces to support bacterial growth. We also used $\mathrm{MWF}^{490}$ medium supplemented with $1 \%$ Casamino Acids (Difco, UK) (MWF+AA). All medium compositions are listed in Dataset 1 and are identical to those used in (47).

Experimental setup. To assemble the resident community, a single isolated colony of each species was selected and inoculated in $10 \mathrm{~mL}$ of Tryptic Soy Broth (TSB) in Erlenmeyer flasks $(50 \mathrm{~mL})$, then incubated overnight at $28^{\circ} \mathrm{C}$ (200 rpm). To achieve exponentially growing bacteria, with a final concentration of $\sim 10^{6}-10^{7} \mathrm{CFU} / \mathrm{mL}$, each bacterial species was inoculated at an $\mathrm{OD}_{600}$ of 0.05 measured by spectrophotometry (Ultrospec 10, Amersham Biosciences), in $20 \mathrm{~mL}$ of TSB in Erlenmeyer flasks $(100 \mathrm{~mL})$ and cultivated 502 at $28^{\circ} \mathrm{C}$, shaken at $200 \mathrm{rpm}$. After $3 \mathrm{~h}, 200 \mu \mathrm{L}$ of each of the ${ }_{504}^{503}$ four resident species were combined and centrifuged (5 min- 505 utes, 10'000 rcf). The bacterial pellet was resuspended in ${ }_{507}^{506}$ $30 \mathrm{~mL}$ of MWF or MWF+AA into borosilicate glass tubes 508 $(16 \times 125 \mathrm{~mm}, 30 \mathrm{~mL})$.

Transfers. All communities (the 4-species or the 3-species resident communities) were incubated at $28^{\circ} \mathrm{C}$ and shaken at $200 \mathrm{rpm}$ for 7 days in either MWF or MWF+AA medium. Every week, $300 \mu \mathrm{L}(1 \%)$ of the week-old culture was trans- ${ }_{512}{ }^{511}$ ferred into fresh medium and the growth cycle repeated. Each ${ }_{513}$ week, we also harvested $1 \mathrm{ml}$ of each culture, spun it down ${ }_{515}^{514}$ at 10,000 rcf for 5 minutes, re-suspended it in glycerol $25 \% 516$ (diluted in PBS) and stocked it at $-80^{\circ} \mathrm{C}$ for future analyses. ${ }_{518}^{517}$ This was repeated for 44 transfers (weeks) to co-evolve the ${ }_{519}$ resident communities or for 4 transfers in the invasion as- ${ }^{520}$ says. The evolutionary experiment was conducted in 5 repli- ${ }^{522}$ cate culture tubes for each condition (3- or 4-species com- ${ }_{524}^{523}$ munity), of which we shown only one here (Fig. S8). After ${ }_{525}^{524}$ the 44 weeks, we isolated one colony of each species, which ${ }^{526}$ we refer to as A. tumefaciens str. MWF431, C. testosteroni ${ }_{528}^{527}$ str. MWF431, M. saperdae str. MWF431 and O. anthropi str. MWF431 for the four-species co-evolved community; and $A$. tumefaciens str. MWF351, C. testosteroni str. MWF351, M. saperdae str. MWF351 and $O$. anthropi str. MWF351 for the 3-species co-evolved community.

Invasion assays. Invasion was performed after 2 days of the first transfer of the resident community. One single colony of each invader species was selected and inoculated in $10 \mathrm{~mL}$ of Tryptic Soy Broth (TSB) in Erlenmeyer flasks $(50 \mathrm{~mL})$ and incubated overnight at $28^{\circ} \mathrm{C}$, shaken at $200 \mathrm{rpm}$. To achieve exponentially growing bacteria, with a final concentration of $\sim 10^{6}-10^{7} \mathrm{CFU} / \mathrm{mL}$, each invader strain was inoculated at an $\mathrm{OD}_{600}$ of 0.05 measured by spectrophotometry (Ultrospec 10, Amersham Biosciences), in 20mL of TSB in Erlenmeyer flasks $(100 \mathrm{~mL})$ and cultivated at $28^{\circ} \mathrm{C}(200 \mathrm{rpm})$. After $3 \mathrm{~h}$, $200 \mu \mathrm{L}$ of the invader species were centrifuged (5 minutes, 10 '000 rcf). The bacterial pellet was resuspended into the same medium of the resident community. $200 \mu \mathrm{L}$ of this suspension were then added to the culture tubes, with or without the resident communities.

Bacterial abundance quantification. The abundance of each resident or invader species was quantified before the inoculation in the MWF or MWF+AA (before combining resident species), and before each transfer using serial dilution and selective plating (Fig. 4). To define invasion outcomes we used an invasion threshold representing the dynamics of an invader species with a growth rate of 0 (its abundance changes only due to dilution, i.e. 100-fold decrease every transfer from the initial population size). By subtracting this threshold value from the abundance of the invader species at transfer 4 , the invasion is defined as successful if $>0$ (the growth rate is positive) or failed if $\leq 0$ (the growth rate is 0 or negative). We used a Kruskal-Wallis test to assess whether effects were significant. We did not used corrections. Why? Raw CFU/mL data and the results of all statistical tests are listed in Dataset 1.

\section{ACKNOWLEDGEMENTS}

We thank Oliver Meacock and Shota Shibasaki for useful and constructive feedback on the manuscript. We thank Christopher van der Gast and lan Thompson for providing the 4 species used to assemble the resident community, and Peter Kuenzi for providing the 4 additional species used as invaders. P.P. and G.A. were funded by the University of Lausanne, S.M. by European Research Council Starting Grant 715097. J.M.A. received funding from the European Union's Horizon 2020 research and innovation programme under grant agreement No. 678841.

\section{References}

1. Madhav P. Thakur, Wim H. van der Putten, Marleen M. P. Cobben, Mark van Kleunen, and Stefan Geisen. Microbial invasions in terrestrial ecosystems. Nature Reviews Microbiology, 17(10):621-631, 2019.

2. H A Mooney and E E Cleland. The evolutionary impact of invasive species. PNAS, 98(10): $5446-5451,2001$.

3. Cyrus Alexander Mallon, Jan Dirk Van Elsas, and Joana Falcão Salles. Microbial invasions: The process, patterns, and mechanisms. Trends in Microbiology, 23(11):719-729, 2015.

4. Siobhán O'Brien, David J Hodgson, and Angus Buckling. Social evolution of toxic metal bioremediation in Pseudomonas aeruginosa. Proceedings of the Royal Society B: Biological Sciences, 281(1787), 2014.

5. Jens Walter, María X. Maldonado-Gómez, and Inés Martínez. To engraft or not to engraft: an ecological framework for gut microbiome modulation with live microbes. Current Opinion in Biotechnology, 49:129-139, 2018.

6. Els van der Goot, Francjan J. van Spronsen, Joana Falcão Salles, and Eddy A. van der Zee. A Microbial Community Ecology Perspective on the Gut-Microbiome-Brain Axis. Frontiers in Endocrinology, 11:611, 2020.

7. Mark Williamson and Alastair Fitter. The varying success of invaders. Ecology, 77(6):1661$1666,1996$. 
bioRxiv preprint doi: https://doi.org/10.1101/2022.03.03.482806; this version posted March 3, 2022. The copyright holder for this preprint (which was not certified by peer review) is the author/funder, who has granted bioRxiv a license to display the preprint in perpetuity. It is made available under aCC-BY 4.0 International license.

\begin{tabular}{|c|c|}
\hline & Target species \\
\hline & $\begin{array}{l}\text { Agrobacterium tumefaciens } \\
\text { Comamonas testosteroni } \\
\text { Microbacterium saperdae } \\
\text { Ochrobactrum anthropi }\end{array}$ \\
\hline & $\begin{array}{l}\text { Aeromonas caviae } \\
\text { Klepsiella pneumoniae } \\
\text { Providencia rettgeri } \\
\text { Pseudomonas fulva }\end{array}$ \\
\hline
\end{tabular}

\begin{tabular}{|l} 
Selective plate \\
LB + sulfamethoxazole $(14.25 \mu \mathrm{g} / \mathrm{mL})$ \\
+ trimethoprim $(0.75 \mu \mathrm{g} / \mathrm{mL})$ \\
$\mathrm{LB}$ \\
$\mathrm{LB}+$ colistin $(10 \mu \mathrm{g} / \mathrm{mL})$ \\
$\mathrm{LB}+$ colistin $(10 \mu \mathrm{g} / \mathrm{mL})$ \\
$\mathrm{MMC}+$ nalidixic acid $(15 \mu \mathrm{g} / \mathrm{mL})$ \\
$\mathrm{KIA}+$ carbenicillin $(50 \mu \mathrm{g} / \mathrm{mL})$ \\
$\mathrm{PIA}+$ tetracycline $(30 \mu \mathrm{g} / \mathrm{mL})$ \\
$+2 \%$ glycerol \\
$\mathrm{CA}+$ carbenicillin $(50 \mu \mathrm{g} / \mathrm{mL})$
\end{tabular}

\begin{tabular}{|l|l|}
\hline Incubation temperature / time & Characteristic \\
$28^{\circ} \mathrm{C} / 48-72 \mathrm{hrs}$ & Green fluorescent (GFP) colonies \\
& \\
$28^{\circ} \mathrm{C} / 24 \mathrm{hrs}$ & White colonies, the first to appear \\
$28^{\circ} \mathrm{C} / 48-72 \mathrm{hrs}$ & Green colonies \\
$28^{\circ} \mathrm{C} / 72-96 \mathrm{hrs}$ & Red fluorescent (mCherry) colonies \\
$37^{\circ} \mathrm{C} / 24 \mathrm{hrs}$ & Dark blue colonies \\
$37^{\circ} \mathrm{C} / 24 \mathrm{hrs}$ & Purple colonies \\
$28^{\circ} \mathrm{C} / 48-72 \mathrm{hrs}$ & White colonies \\
$37^{\circ} \mathrm{C} / 24 \mathrm{hrs}$ & \\
\end{tabular}

Fig. 4. Selective plates list

8. Jane A. Catford, Roland Jansson, and Christer Nilsson. Reducing redundancy in invasion 596 ecology by integrating hypotheses into a single theoretical framework. Diversity and Distri- 597 butions, 15(1):22-40, 2009.

9. Daniel Simberloff. The Role of Propagule Pressure in Biological Invasions. Annual Review 599 of Ecology, Evolution, and Systematics, 40(1):81-102, 2009. Dynamics of an experimental microbial invasion. PNAS, 112(37):11594-11599, 2015.602

11. J. N. Barney, M. W. Ho, and D. Z. Atwater. Propagule pressure cannot always overcome 603 biotic resistance: The role of density-dependent establishment in four invasive species. 604 Weed Research, 56(3):208-218, 2016.

12. T. Ketola, K. Saarinen, and L. Lindström. Propagule pressure increase and phylogenetic 606 diversity decrease community's susceptibility to invasion. BMC Ecology, 17(1):1-7, 2017. 607

13. Jean C.C. Vila, Matt L. Jones, Matishalin Patel, Tom Bell, and James Rosindell. Uncovering 608 the rules of microbial community invasions. Nature Ecology and Evolution, 3(8):1162-1171, 609 2019.

14. Michael D. Dressler, Josue Conde, Omar Tonsi Eldakar, and Robert P. Smith. Timing be- 61 tween successive introduction events determines establishment success in bacteria with an 612 Allee effect. Proceedings of the Royal Society B: Biological Sciences, 286(1902):20190598, 613 2019.

15. Matt Lloyd Jones, Damian William Rivett, Alberto Pascual-Garria, and Thomas Bell. Re- 615 lationships between community composition, productivity and invasion resistance in semi- 616 natural bacterial microcosms. eLife, 10, 2021.

16. Damian W. Rivett, Matt L. Jones, Josep Ramoneda, Shorok B. Mombrikotb, Emma Ran- 618 some, and Thomas Bell. Elevated success of multispecies bacterial invasions impacts com- 619 munity composition during ecological succession. Ecology Letters, 21(4):516-524, 2018. 620

17. Ted $\mathrm{J}$ Case. Invasion resistance arises in strongly interacting species-rich model competition 621 communities. PNAS, 87(24):9610-9614, 1990.

18. Alexandre Jousset, Wiebke Schulz, Stefan Scheu, and Nico Eisenhauer. Intraspecific geno- 623 typic richness and relatedness predict the invasibility of microbial communities. ISME Jour- 624 nal, 5(7):1108-1114, 2011.

19. Nico Eisenhauer, Stefan Scheu, and Alexandre Jousset. Bacterial diversity stabilizes com- 626 munity productivity. PLOS ONE, 7(3), 2012.

20. Zhong Wei, Tianjie Yang, Ville Petri Friman, Yangchun Xu, Qirong Shen, and Alexandre 628 Jousset. Trophic network architecture of root-associated bacterial communities determines 629 pathogen invasion and plant health. Nature Communications, 6:8413, 2015.

21. Stefano Amalfitano, Manuela Coci, Gianluca Corno, and Gian Marco Luna. A microbial 631 perspective on biological invasions in aquatic ecosystems. Hydrobiologia, 746(1):13-22, 632 2015.

22. Shao Peng Li, Jiagi Tan, Xian Yang, Chao Ma, and Lin Jiang. Niche and fitness differences 634 determine invasion success and impact in laboratory bacterial communities. ISME Journal, 635 13(2):402-412, 2019.

23. Michael Baumgartner, Katia R. Pfrunder-Cardozo, and Alex R. Hall. Microbial community 637 composition interacts with local abiotic conditions to drive colonization resistance in human 638 gut microbiome samples. Proceedings of the Royal Society B: Biological Sciences, 288639 (1947), 2021.

24. Helen M. Kurkjian, M. Javad Akbari, and Babak Momeni. The impact of interactions on in- 641 vasion and colonization resistance in microbial communities. PLOS Computational Biology, 642 17(1):e1008643, 2021.

25. David Tilman. Niche tradeoffs, neutrality, and community structure: A stochastic theory of 644 resource competition, invasion, and community assembly. PNAS, 101(30):10854-10861, 645 2004.

26. Jan Dirk Van Elsas, Mario Chiurazzi, Cyrus A Mallon, Dana Elhottova, Václav Krištůfek, 647 and Joana Falcão Salles. Microbial diversity determines the invasion of soil by a bacterial 648 pathogen. PNAS, 109(4):1159-1164, 2012

27. Kevin R Foster and Thomas Bell. Competition, Not Cooperation, Dominates Interactions 650 among Culturable Microbial Species. Current biology, 22(19):1845-50, 2012.

28. Sara Mitri and Kevin Richard Foster. The Genotypic View of Social Interactions in Microbial 652 Communities. 2013.

29. Jared Kehe, Anthony Ortiz, Anthony Kulesa, Jeff Gore, Paul C. Blainey, and Jonathan Fried- 654 man. Positive interactions are common among culturable bacteria. Science Advances, 7655 (45): 7159,2021

30. Joseph H. Connell and Ralph O. Slatyer. Mechanisms of Succession in Natural Communi- 657 ties and Their Role in Community Stability and Organization. The American Naturalist, 111658 (982):1119-1144, 1977.

31. Reena Debray, Robin A. Herbert, Alexander L. Jaffe, Alexander Crits-Christoph, Mary E. 660 Power, and Britt Koskella. Priority effects in microbiome assembly. Nature Reviews Micro- 661 biology, pages 1-13, 2021.
32. Manoshi S. Datta, Elzbieta Sliwerska, Jeff Gore, Martin F. Polz, and Otto X. Cordero. Microbial interactions lead to rapid micro-scale successions on model marine particles. Nature Communications, 7:11965, 2016.

33. Tim N. Enke, Manoshi S. Datta, Julia Schwartzman, Nathan Cermak, Désirée Schmitz, Julien Barrere, Alberto Pascual-García, and Otto X. Cordero. Modular Assembly of Polysaccharide-Degrading Marine Microbial Communities. Current Biology, 29(9):15281535.e6, 2019

34. Varun Mazumdar, Salomon Amar, and Daniel Segrè. Metabolic Proximity in the Order of Colonization of a Microbial Community. PLOS ONE, 8(10):e77617, 2013.

35. Matti Gralka, Rachel Szabo, Roman Stocker, and Otto X. Cordero. Trophic Interactions and the Drivers of Microbial Community Assembly. Current Biology, 30(19):R1176-R1188, 2020.

36. Alexander H Rickard, Peter Gilbert, Nicola J High, Paul E Kolenbrander, and Pauline S Handley. Bacterial coaggregation: an integral process in the development of multi-species biofilms. Trends in Microbiology, 11(2):94-100, 2003.

37. Paul E. Kolenbrander, Robert J. Palmer, Saravanan Periasamy, and Nicholas S. Jakubovics. Oral multispecies biofilm development and the key role of cell-cell distance. Nature Reviews Microbiology 2010 8:7, 8(7):471-480, 2010.

38. J. M. Monier and S. E. Lindow. Aggregates of resident bacteria facilitate survival of immigrant bacteria on leaf surfaces. Microbial Ecology, 49(3):343-352, 2005.

39. Cesar Poza-Carrion, Trevor Suslow, and Steven Lindow. Resident Bacteria on Leaves Enhance Survival of Immigrant Cells of Salmonella enterica. Phytopathology, 103(4):341-351, 2013.

40. Mei Li, Zhong Wei, Jianing Wang, Alexandre Jousset, Ville Petri Friman, Yangchun Xu, Qirong Shen, and Thomas Pommier. Facilitation promotes invasions in plant-associated microbial communities. Ecology Letters, 22(1):149-158, 2019.

41. Sylvie Estrela, Jean C.C. Vila, Nanxi Lu, Djordje Bajić, Maria Rebolleda-Gómez, Chang-Yu Chang, Joshua E. Goldford, Alicia Sanchez-Gorostiaga, and Álvaro Sánchez. Functional attractors in microbial community assembly. Cell Systems, 13(1):29-42.e7, 2022.

42. Mark D. Bertness and Ragan Callaway. Positive interactions in communities. Trends in Ecology Evolution, 9(5):191-193, 1994

43. Jesse R. Zaneveld, Ryan McMinds, and Rebecca Vega Thurber. Stress and stability: applying the Anna Karenina principle to animal microbiomes. Nature Microbiology 2017 2:9, 2 (9):1-8, 2017.

44. Franciska T. de Vries, Rob I. Griffiths, Mark Bailey, Hayley Craig, Mariangela Girlanda, Hyun Soon Gweon, Sara Hallin, Aurore Kaisermann, Aidan M. Keith, Marina Kretzschmar, Philippe Lemanceau, Erica Lumini, Kelly E. Mason, Anna Oliver, Nick Ostle, James I. Prosser, Cecile Thion, Bruce Thomson, and Richard D. Bardgett. Soil bacterial networks are less stable under drought than fungal networks. Nature Communications, 9(1), 2018.

45. Joshua E. Goldford, Nanxi Lu, Djordje Bajić, Sylvie Estrela, Mikhail Tikhonov, Alicia Sanchez-Gorostiaga, Daniel Segrè, Pankaj Mehta, and Alvaro Sanchez. Emergent simplicity in microbial community assembly. Science, 361(6401):469-474, 2018.

46. Linh T.T. Nguyen, Katie Broughton, Yui Osanai, lan C. Anderson, Michael P. Bange, David T. Tissue, and Brajesh K. Singh. Effects of elevated temperature and elevated CO 2 on soil nitrification and ammonia-oxidizing microbial communities in field-grown crop. Science of the Total Environment, 675:81-89, 2019.

47. Philippe Piccardi, Björn Vessman, and Sara Mitri. Toxicity drives facilitation between 4 bacterial species. PNAS, 116(32):15979-15984, 2019.

48. Damian J. Hernandez, Aaron S. David, Eric S. Menges, Christopher A. Searcy, and Michelle E. Afkhami. Environmental stress destabilizes microbial networks. ISME Journal, 15(6):1722-1734, 2021.

49. Mark C. Urban and Luc De Meester. Community monopolization: Local adaptation enhances priority effects in an evolving metacommunity. Proceedings of the Royal Society B: Biological Sciences, 276(1676):4129-4138, 2009.

50. Joost Vanoverbeke, Mark C. Urban, and Luc De Meester. Community assembly is a race between immigration and adaptation: eco-evolutionary interactions across spatial scales. Ecography, 39(9):858-870, 2016.

51. Luc De Meester, Joost Vanoverbeke, Laurens J. Kilsdonk, and Mark C. Urban. Evolving Perspectives on Monopolization and Priority Effects. Trends in Ecology and Evolution, 31 (2): 136-146, 2016

52. Nicolas Loeuille and Mathew A. Leibold. Evolution in metacommunities: On the relative importance of species sorting and monopolization in structuring communities. American Naturalist, 171(6):788-799, 2008.

53. Christopher P. Nadeau, Timothy E. Farkas, Andrea M. Makkay, R. Thane Papke, and Mark C. Urban. Adaptation reduces competitive dominance and alters community assembly. Proceedings of the Royal Society B: Biological Sciences, 288(1945), 2021.

54. Christopher J van der Gast and lan $\mathrm{P}$ Thompson. Effects of $\mathrm{pH}$ amendment on metal work- 
bioRxiv preprint doi: https://doi.org/10.1101/2022.03.03.482806; this version posted March 3, 2022. The copyright holder for this preprint (which was not certified by peer review) is the author/funder, who has granted bioRxiv a license to display the preprint in perpetuity. It is made available under aCC-BY 4.0 International license.

ing fluid wastewater biological treatment using a defined bacterial consortium. Biotechnology and Bioengineering, 89(3):357-66, 2005.

55. Christoph Ratzke, Julien Barrere, and Jeff Gore. Strength of species interactions determines biodiversity and stability in microbial communities. Nature Ecology and Evolution, 4 (3):376-383, 2020.

56. Tim N. Enke, Gabriel E. Leventhal, Matthew Metzger, José T. Saavedra, and Otto X Cordero. Microscale ecology regulates particulate organic matter turnover in model marine microbial communities. Nature Communications, 9(1):2743, 2018.

57. Ori Furman, Liat Shenhav, Goor Sasson, Fotini Kokou, Hen Honig, Shamay Jacoby, Tomer Hertz, Otto X. Cordero, Eran Halperin, and Itzhak Mizrahi. Stochasticity constrained by deterministic effects of diet and age drive rumen microbiome assembly dynamics. Nature Communications 2020 11:1, 11(1):1-13, 2020.

58. Katharine Z. Coyte, Chitong Rao, Seth Rakoff-Nahoum, and Kevin R. Foster. Ecological rules for the assembly of microbiome communities. PLoS Biology, 19(2), 2021.

59. Natalia Molinero, Lorena Ruiz, Borja Sánchez, Abelardo Margolles, and Susana Delgado. Intestinal Bacteria Interplay With Bile and Cholesterol Metabolism: Implications on Host Physiology. Frontiers in Physiology, 10, 2019.

60. Lorena Ruiz, Abelardo Margolles, and Borja Sánchez. Bile resistance mechanisms in Lactobacillus and Bifidobacterium. Frontiers in Microbiology, 4, 2013.

61. Philippe Gérard. Metabolism of Cholesterol and Bile Acids by the Gut Microbiota. Pathogens 2014, Vol. 3, Pages 14-24, 3(1):14-24, 2013.

62. Kapil Amarnath, Avaneesh V. Narla, Sammy Pontrelli, Jiajia Dong, Tolga Caglar, Brian R Taylor, Julia Schwartzman, Uwe Sauer, Otto X. Cordero, and Terence Hwa. Stress-induced cross-feeding of internal metabolites provides a dynamic mechanism of microbial cooperation. bioRxiv, page 2021.06.24.449802, 2021.

63. Damian W Rivett, Thomas Scheuerl, Christopher T Culbert, Shorok B Mombrikotb, Emma Johnstone, Timothy G Barraclough, and Thomas Bell. Resource-dependent attenuation of species interactions during bacterial succession. The ISME Journal, 10:2259-2268, 2016.

64. K. L. Cook, J. L. Garland, A. C. Layton, H. M. Dionisi, L. H. Levine, and G. S. Sayler. Effect of microbial species richness on community stability and community function in a mode plant-based wastewater processing system. Microbial Ecology, 52(4):725-737, 2006.

65. Yuki Miura, Mirian Noriko Hiraiwa, Tsukasa Ito, Takanori Itonaga, Yoshimasa Watanabe, and Satoshi Okabe. Bacterial community structures in MBRs treating municipal wastewater: Relationship between community stability and reactor performance. Water Research, 41(3): 627-637, 2007.

66. Stefano Allesina and Si Tang. Stability criteria for complex ecosystems. Nature, 483(7388) 205-208, 2012.

67. Oylum Erkus, Victor C.L. De Jager, Maclej Spus, Ingrid J. Van Alen-Boerrigter, Irma M.H. Van Rijswijck, Lucie Hazelwood, Patrick W.M. Janssen, Sacha A.F.T. Van Hijum, Michiel Kleerebezem, and Eddy J. Smid. Multifactorial diversity sustains microbial community stability. ISME Journal, 7(11):2126-2136, 2013.

68. Thomas Bell, Jonathan A. Newman, Bernard W. Silverman, Sarah L. Turner, and Andrew K. Lilley. The contribution of species richness and composition to bacterial services. Nature, 436(7054):1157-1160, 2005.

69. Tadashi Fukami. Historical Contingency in Community Assembly: Integrating Niches, Species Pools, and Priority Effects. Annual Review of Ecology, Evolution, and Systematics, 46(1):1-23, 2015. 